Ann. Génét. Sél. anim., I97 I, 3 (1), 5-I5.

\title{
AGGLUTINOGÈNE « Hi » ET PERFORMANCES CHEZ LA POULE
}

\author{
I. DURAND et P. MÉRAT \\ Laboratoive de Génétique factorielle, \\ Centre national de Recherches zootechniques, INRA \\ 78-Jouy-en-Josas
}

\section{RÉSUMÉ}

Ayant observé dans notre troupeau de poules un polymorphisme pour un agglutinogène présent sur les hématies et décelé par des extraits de graines (Lens culinaris), nous avons vérifié que sa transmission est celle d'un gène simple et de son allèle récessif, la présence de l'agglutinogène étant dominante. En raison de ses caractéristiques, nous désignons ce gène par le symbole $\mathrm{Hi}$ déjà connu (Scheinberg et Reckel, 1961).

Nous avons recherché, d'autre part, les différences quantitatives pouvant être associées à ce facteur. Dans notre population, les poules "positives" ( $H i / H i$ ou $H i / h i)$ produisent un nombre d'œufs de 8,5 p. 100 plus élevé que les "négatives " $(h i / h i)$ pendant les premiers mois de ponte $(\mathrm{P}<0,001)$. Ceci ne peut raisonnablement être attribué à la non-expression de l'agglutinogène chez des femelles ne pondant pas et doit donc correspondre à une aifférence réelle entre les génotypes comparés. En outre, la variance du nombre d'œufs et de l'âge au premier œuf est moindre pour les poules "positives " $(P<0,01)$, l'épaisseur des coquilles de leurs cufs est un peu supérieure et moins variable $(P<0,05)$, l'albumen moins consistant $(P<0,001)$. Enfin, leur poids adulte est légèrement inférieur $(\mathrm{P}<0,05)$. Par contre, il n'y a pas de différence de mortalité entre animaux des deux phénotypes.

Une comparaison, par voie indirecte, des génotypes $\mathrm{Hi} / \mathrm{Hi}$ et $\mathrm{Hi} / \mathrm{hi}$ suggère une supériorité de l'hétérozygote sur les deux homozygotes pour le nombre d'œufs pondus.

\section{INTRODUCTION}

SCHEINBERG et RECKEL ( $196 \mathrm{I} a$ et $b$ ) ont mis en évidence dans diverses populations chez la Poule un agglutinogène décelable par certains extraits végétaux, de légumineuses en particulier (Pisum sativum, Lathyrus cicera). La présence de cet agglutinogène correspond apparemment à un facteur mendélien simple dominant, dénommé $H i$ par ces auteurs (SchEINBERG et RECKEI, rg62). Ce facteur se manifeste seulement chez les poules en ponte, un taux suffisant d'œstrogènes dans le sang étant nécessaire à son expression (SCHEINBERG et RECKEL, I96Ic, I962). 
Un polymorphisme a été constaté dans d'autres populations (par exemple BOREL, I962, I964; Mitsumoto, I964; ERdös, I969). D'après BorEL, le même agglutinogène $H i$ est décelé par les lentilles (Lens culinaris) et les vesces (Vicia sativa, Vicia cracca).

On ne possède pratiquement pas de renseignements sur les associations possibles de ce facteur avec les performances quantitatives des animaux. Seuls, à notre connaissance, PETrovsky et al. (I968) indiquent que, dans quatre lignées de Leghorns étudiées sur deux ans, les poules n'extériorisant pas le facteur $H i$ lors d'un test fait après dix mois de ponte avaient en moyenne une production supérieure. Ils semblent conclure à une corrélation physiologique avec l'état de ponte, et non à une association de nature génétique.

Ayant retrouvé dans notre troupeau un polymorphisme analogue à ceux décrits par les auteurs précédents, nous avons voulu, après avoir vérifié de façon complète le caractère mendélien de sa transmission, chercher l'existence de liaisons avec des caractéristiques quantitatives d'intérêt zootechnique.

\section{MATÉRIEL ET MÉTHODES}

La population étudiée, issue d'un croisement de plusieurs races fait en 1954 , contient un certain nombre de gènes " marqueurs " en ségrégation (MÉrAT, 1967). Les premières analyses faites en 1966 y ont révélé un polymorphisme pour le facteur " $H i$ ". Par la suite, ce polymorphisme a été maintenu par le choix des reproducteurs relativement à ce facteur, lorsque ce choix était possible.

Cette population est subdivisée en deux troupeaux, reproduits en pedigree sans consanguinité systématique, l'un au printemps, l'autre à l'automne.

Les jeunes sont élevés au sol, les pondeuses également, sauf en 1969 où elles étaient en cages depuis l'âge de 15 semaines. Les caractères quantitatifs considérés ici sont le poids à 8 semaines, l'âge à la ponte du premier œuf, le nombre d'œufs pondus par les poulettes vivantes en fin de contrôle (à 10 mois d'âge environ), leur poids, le poids moyen des œufs sur deux semaines consécutives, l'épaisseur de leur coquille et la hauteur de l'albumen à ce même âge. Ces deux derniers caractères étaient évalués sur 2 œufs consécutifs par poule : l'épaisseur de la coquille (y compris la membrane) est mesurée au 1 /100 de mm vers l'équateur de l'œuf par un comparateur, la hauteur de l'albumen épais notée au 1/10 de mm grâce à un micromètre à trépied sur l'œuf cassé et étalé sur une surface vitrée plane. Les nombres d'œufs utilisés par poule pour ces deux dernières mesures seraient trop faibles pour une estimation précise d'une valeur individuelle, mais ils suffisent à la comparaison de groupes.

La mortalité en poussinière (jusqu'à 8 semaines et ultérieure) a également été comparée, cette fois pour la descendance des mères $(H i)$ et $(h i)$, les individus n'étant testés pour $H i$ que vers la fin de la période de contrôle de leurs performances.

Les résultats analysés vont de 1966 à 1969 inclus, pour les deux troupeaux élevés chaque année (1969 n'incluant que le troupeau né au printemps).

\section{Identification des phénotypes}

Les animaux sont testés avec un extrait de lentilles (Lens culinaris), variété "verte ", la variété "blonde " ne donnant pas de résultats satisfaisants. Le mode de préparation de l'extrait et la réaction avec le sang sont conformes aux descriptions de Scheinberg et RECKEL (1961a) et de Borel (1962).

Les lentilles sont broyées dans un mortier après macération pendant vingt-quatre heures dans du sérum physiologique additionné de 4 p. 1000 de merthiolate de soude afin de prévenir toute fermentation. Le jus est extrait au moyen d'une presse de laboratoire, purifié par décantation et centrifugation et conservé au congélateur à $-20^{\circ} \mathrm{C}$.

Les réactions se font en additionnant 1 goutte de suspension d'hématies à 2 p. 100 préalablement lavées, à 2 gouttes d'extrait, ce dernier étant dilué à $1 / 2$. Les tubes sont centrifugés à 500 tours pendant une minute. Les lectures sont faites aussitôt la centrifugation, puis vérifiées une heure après, certaines réactions ne se produisant pas immédiatement. Toutes les manipulations se font à la température de $24^{\circ} \mathrm{C}$ environ. 
Les réactions sont suffisamment précises pour être lues à l'œil nu. Une loupe est utilisée en cas de doute. Le nombre de cas litigieux dans nos conditions est relativement très faible.

Le test a lieu vers 8 mois d'âge. La très grande majorité des poules est alors en ponte et la plupart des non-réagissantes peuvent donc être considérées comme étant du génotype hi/hi. Pour toutes les poules "négatives " dont le génotype n'était pas connu de par leur ascendance, et présentant quelque doute quant à la lecture de la réaction d'agglutination ou du fait qu'elles n'étaient pas en ponte à une date rapprochée de l'analyse, un second test est refait environ deux mois après. De cette façon, l'incertitude sur la présence du gène $H i$ est restreinte à un nombre trop faible de poules pour influer de façon appréciable sur les résultats. De plus, le petit nombre de poules hors de ponte à l'époque des deux tests n'est pas pris en considération dans le présent travail.

\section{Analyse des résultats}

Les proportions mendéliennes sont testées pour leur conformité aux proportions théoriques prévues, après élimination des familles de sœurs dont l'effectif est très faible.

Les caractères quantitatifs sont ensuite comparés pour les 2 phénotypes $(H i)$ et $(h i)$, à l'intérieur des familles présentant une ségrégation pour le facteur correspondant. La comparaison des moyennes est faite à partir de couples de sœurs élevées ensemble, écloses le même jour en ce qui concerne la comparaison relative au poids à 8 semaines, ou au maximum à 15 jours d'intervalle pour les autres comparaisons. Pour la mortalité, tous les animaux de familles en ségrégation au locus $H i$ sont pris en considération et un test de $\chi^{2}$ est fait sur l'ensemble des générations (qui différaient relativement peu pour le taux global de mortalité).

Nous avons enfin cherché à comparer entre eux par voie indirecte les génotypes $\mathrm{Hi} H i$ et $\mathrm{Hi} \mathrm{hi,} \mathrm{puis} \mathrm{les} \mathrm{descendances} \mathrm{de} \mathrm{croisements} \mathrm{réciproques;} \mathrm{les} \mathrm{détails} \mathrm{de} \mathrm{cette} \mathrm{comparaison} \mathrm{sont}$ contenus dans le paragraphe correspondant.

\section{RÉSULTATS}

\section{I. - Mode de transmission héréditaire}

Le tableau I indique, sur l'ensemble des données, les proportions de phénotypes $(H i)$ et $(h i)$ obtenus dans la descendance femelle à l'époque du test suivant le génotype supposé des parents. Ce dernier est déduit de l'examen de la descendance dans le cas des " mâles "; un coq ayant eu des filles réagissantes (Hi) avec des mères non-réagissantes $(h i)$ est considéré comme homozygoté dominant ou hétérozygote suivant les proportions dans la descendance.

\section{TABLEAU I}

Proportions mendéliennes suivant le génotype supposé des parents

\begin{tabular}{|c|c|c|c|c|c|}
\hline \multirow{2}{*}{$\begin{array}{c}\text { Génotype supposé } \\
\text { des parents }\end{array}$} & \multicolumn{2}{|c|}{ Nombre de filles } & \multirow{2}{*}{$\begin{array}{l}\text { Proportion } \\
\text { théorique } \\
\text { du phénotype } \\
(H i) \%\end{array}$} & \multirow{2}{*}{$x^{2}$} & \multirow{2}{*}{ Probabilité } \\
\hline & $(H i)$ & (hi) & & & \\
\hline ๙ $H i H i \times q \begin{cases}H i & h i \\
h i & h i\end{cases}$ & I I 2 & o & IOO & 一 & - \\
\hline o Hihi $\times$ 우 Hihi & 368 & 145 & 75 & 2,92 & N.S. \\
\hline o Hihi $\times$ o $h i h i$ & 260 & $25^{\circ}$ & 50 & 0,20 & $>0,80$ \\
\hline o hi hi $\quad$ × $+H i h i$ & 76 & 87 & $5^{\circ}$ & 0,74 & $>0,50$ \\
\hline o hi hi $\times$ q hi hi & 0 & 295 & o & 一 & - \\
\hline
\end{tabular}


Dans l'ensemble, ces proportions confirment bien l'hypothèse d'un gène autosomal à deux allèles, $H i$ étant dominant sur $h i$. Le fait que le croisement de deux parents non réagissants donne uniquement des enfants non réagissants indique en outre que notre identification des génotypes doit être satisfaisante. I1 en est de même des proportions obtenues lorsqu'un parent est supposé hétérozygote et 1'autre homozygote récessif. Quant au croisement de deux hétérozygotes, il ne manifeste pas non plus d'écart significatif par rapport à la proportion attendue. On note cependant un léger défaut de descendants réagissants : il est vraisemblable que cela provient de quelques familles ne comportant que des réagissants, et non comptés de ce fait, la mère étant en réalité $H i h i$ sans qu'il soit possible de le déceler. Un léger biais dans le sens observé est ainsi prévisible.

\section{2. - Comparaison des phénotypes (Hi) et (hi) pour des performances quantitatives}

Le tableau 2 présente la comparaison des valeurs moyennes et des variances des femelles de phénotype $(H i)$ - génotype $H i H i$ ou $H i$ hi - et de phénotype $(h i)$ - génotype $h i h i$ - pour les 7 caractères quantitatifs retenus pour cette étude.

Les poules $h i$ hi ont un poids adulte légèrement supérieur (de $40 \mathrm{~g}$, la différence étant significative au seuil $5 \%$ ). Une différence non significative, mais de même sens, est suggérée pour le poids à 8 semaines ( + I I g) et le poids des œufs ( $+0,3 \mathrm{~g}$ ).

Par contre, on constate une supériorité très nette du phénotype $(H i)$ pour le nombre d'œufs produits pendant les premiers mois de ponte. La différence, significative au seuil I p. I ooo, est de 8,5 p. Ioo en faveur des poules réagissantes. Elle est de même sens dans chacune des 7 générations étudiées. De plus, la variance est significativement inférieure pour les poules $(H i)(\mathrm{P}<0, \mathrm{or})$, et il en est de même de la variance de l'âge au premier œuf, la différence moyenne pour ce caractère, quant à elle, étant faible (I jour) et non significative.

Enfin, 1'épaisseur des coquilles d'œufs est un peu supérieure et moins variable pour les poules $(H i)(\mathrm{P}<0,05)$, alors qu'au contraire la hauteur de l'albumen de 1 'œuf cassé est très sensiblement inférieure $(\mathrm{P}<0,00 \mathrm{I})$. Une correction pour le poids de l'œuf n'a pas été jugée nécessaire pour ce dernier caractère, étant donnée la différence faible et non significative entre les deux phénotypes pour ce poids.

Nous avons tenté de vérifier si la différence de production d'œufs pouvait s'expliquer par une différence de longueur moyenne des séries de ponte, en mesurant cette longueur sur les poules des deux phénotypes gardés chaque année comme reproductrices pendant leur période de reproduction. Cet échantillon n'avait pas été soumis à une très forte sélection relativement à l'intensité de ponte antérieure. Au total, pour 203 reproductrices $(H i)$ et $273(h i)$, la longueur moyenne des séries est respectivement 2,37 et 2,43 œufs et la différence n'est pas significative $(\mathrm{t}=-0,53)$.

Pour la mortalité, comme indiqué plus haut, il n'était possible de comparer que la descendance des mères $(H i)$ à celles des mères $(h i)$ avec des pères d'un même 


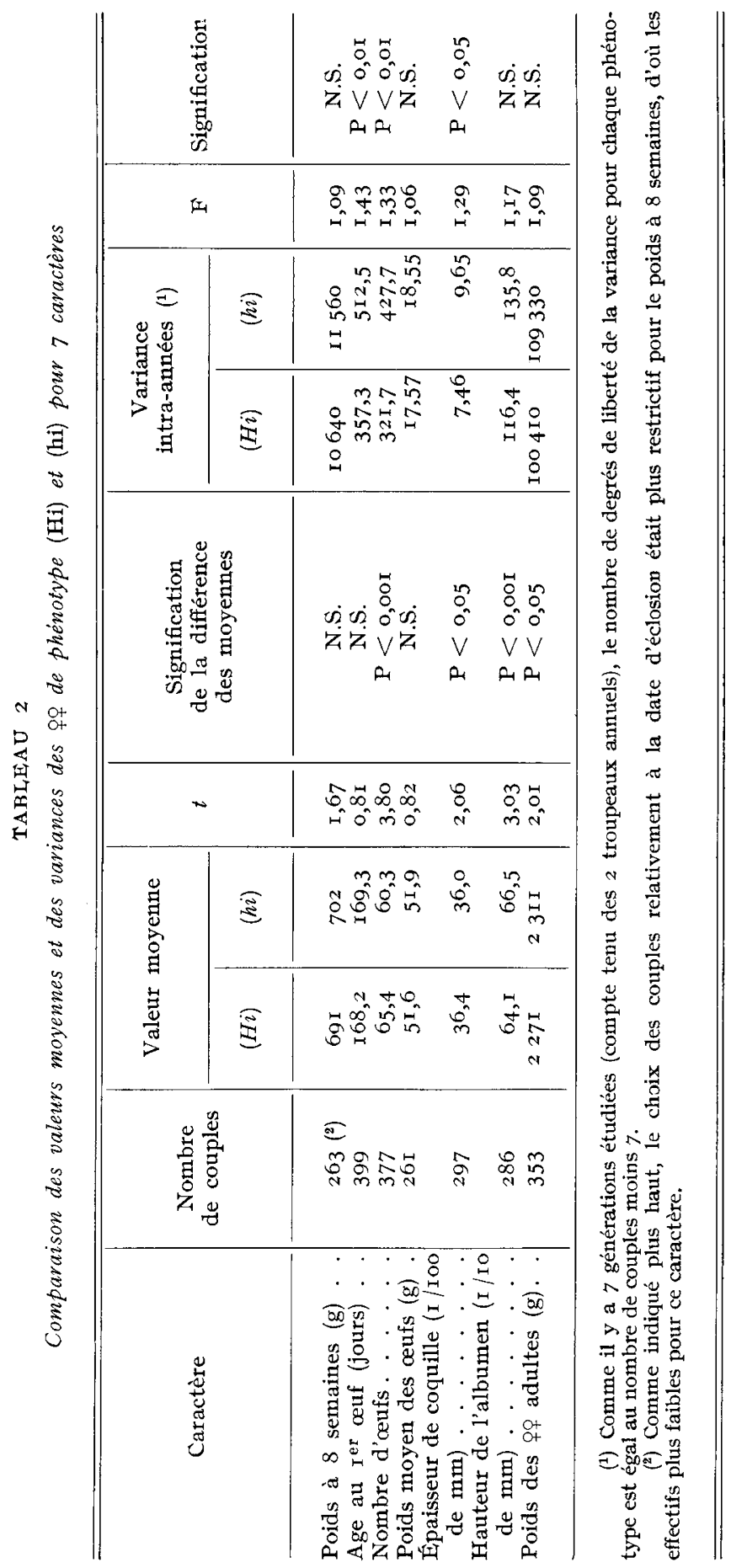


génotype, $H i$ hi, la proportion des deux phénotypes dans les deux groupes de descendances étant différente. Le tableau 3 indique qu'il n'y a aucune différence de mortalité, soit avant, soit après l'âge de 8 semaines. Étant donné les effectifs disponibles, un $\chi^{2}$ ne pouvait être calculé que sur l'ensemble des générations étudiées. Comme nous l'avons mentionné, ceci était rendu licite par l'absence de différences importantes du taux de mortalité d'une génération à l'autre.

TABIEAU 3

Mortalité comparée pour la descendance de mères (Hi) ou (hi) accouplées à des pères d'un même génotype (Hi hi)

\begin{tabular}{|c|c|c|c|c|c|c|}
\hline \multirow[b]{2}{*}{ Age } & \multicolumn{2}{|c|}{ Mères $(H i)$} & \multicolumn{2}{|c|}{ Mères $(h i)$} & \multicolumn{2}{|c|}{ Test de la différence } \\
\hline & $\begin{array}{c}\text { Nombre } \\
\text { de descendants } \\
\text { au départ }\end{array}$ & $\begin{array}{c}\text { Mortalité } \\
(\%)\end{array}$ & $\begin{array}{c}\text { Nombre } \\
\text { de descendants } \\
\text { au départ }\end{array}$ & $\begin{array}{c}\text { Mortalité } \\
(\%)\end{array}$ & $x^{2}$ & Signification \\
\hline $\begin{array}{lll}\text { Avant } 8 & \text { sem. } \\
\text { Après } 8 & \text { sem. }\end{array}$ & $\begin{array}{r}\text { I } 393 \\
374\end{array}$ & $\begin{array}{r}4,80 \\
\text { I I }, 76\end{array}$ & $\begin{array}{r}1038 \\
479\end{array}$ & $\begin{array}{r}3,85 \\
13,15\end{array}$ & $\begin{array}{l}\mathbf{I}, 29 \\
0,37\end{array}$ & $\begin{array}{l}\text { N.S. } \\
\text { N.S. }\end{array}$ \\
\hline
\end{tabular}

\section{3. - Comparaison des génotypes $\mathrm{Hi} \mathrm{Hi}$ et $\mathrm{Hi}$ hi}

I1 n'était pas possible, pratiquement, de comparer directement les performances d'un échantillon suffisant de if de ces deux génotypes, mais une indication indirecte était donnée par la moyenne des descendances de mères de génotype vérifié $H i H i$ ou $H i$ hi avec des pères d'un même génotype. En l'occurrence, la possibilité se présentait avec des pères $H i$ hi. La descendance des deux types de mères comporte alors, parmi les phénotypes $(H i)$, une proportion théorique de moitié de génotypes $H i$ hi pour les mères $H i H i$, et $2 / 3$ pour les mères $H i h i$.

Le tableau 4 montre, sur l'ensemble des années étudiées, les performances moyennes des filles de mères des deux génotypes, limitées aux enfants de phénotype $(H i)$ et aux générations contenant les deux catégories de mères.

Les différences sont relativement peu importantes et non significatives, sauf pour le nombre d'œufs pondus, pour lequel la descendance des mères $H i$ hi présente une supériorité moyenne de Ir,8 p. Ioo. Pour ce critère, le tableau 5 donne l'analyse de variance, sur les moyennes de mères, avec les facteurs contrôlés génotype de la mère et génération (I), la méthode suivie étant celle de SNEDECOR (I959) pour le cas de nombres variables dans les sous-classes et la variance "interaction " n'ayant pas été calculée exactement.

( ${ }^{1}$ Avec plusieurs mères par père, on n'avait pas indépendance totale entre les différentes mères. mais les effectifs étaient insuffisants pour regrouper les données par pères. 
TABLEAU 4

Comparaison de la descendance de mères $\mathrm{Hi} \mathrm{Hi}$ et $\mathrm{Hi}$ hi accouplées à des pères du même génotype ( $\mathrm{Hi} \mathrm{hi)}$

\begin{tabular}{|c|c|c|c|c|}
\hline \multirow{2}{*}{ Caractère } & \multicolumn{2}{|c|}{ Nombre de mères } & \multicolumn{2}{|c|}{$\begin{array}{l}\text { Valeur moyenne } \\
\text { de la descendance }\end{array}$} \\
\hline & $\mathrm{Hi} \mathrm{Hi}$ & $H i$ hi & Mères $H i \quad H i$ & Mères $H i h i$ \\
\hline Poids à 8 semaines des Oे $^{*}(\mathrm{~g})$. & I 8 & 66 & 805 & 802 \\
\hline Poids à 8 semaines des q⿱ $(\mathrm{g})$. & I 8 & 66 & 682 & 665 \\
\hline Age au I $^{\text {er }}$ œuf $(\mathrm{j}) . \ldots$. & I 8 & 65 & I 72,2 & $\mathrm{I} 69,8$ \\
\hline Nombre d'œufs. . . & I 8 & 65 & $6 \mathrm{I}, \mathrm{O}$ & 68,2 \\
\hline Poids moyen des oufs (g). . . . . & I 8 & $6 \mathrm{I}$ & 52,5 & $5 \mathrm{I}, 8$ \\
\hline Épaisseur de coquille (I/Ioo de $\mathrm{mm}$ ) & I 8 & $6 \mathrm{I}$ & $37, \mathrm{I}$ & 37,1 \\
\hline Hauteur de l'albumen (I/ro de $\mathrm{mm}$ ) & I 8 & 60 & 67,2 & 67,3 \\
\hline Poids des PO adultes $(\mathrm{g}) . \ldots$ & I 8 & 65 & 2310 & 2293 \\
\hline
\end{tabular}

TABLEAU 5

Analyse de variance du nombre d'outs sur les moyennes de mères, suivant le génotype maternel pour $\mathrm{Hi}$ et la génération

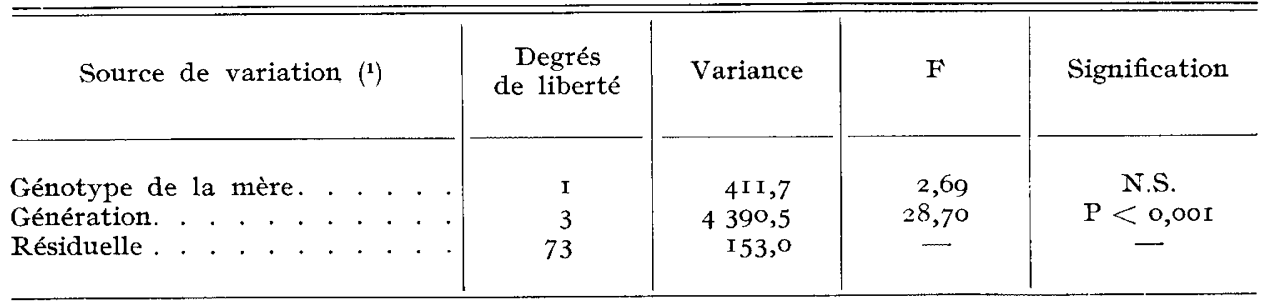

(1) Variance "interaction " non calculée exactement.

La différence entre descendances des mères des deux génotypes, quoique plus importante en valeur absolue que dans la comparaison entre individus de phénotype $(H i)$ et $(h i)$, n'atteint pas tout à fait le seuil 5 p. Ioo de signification, du fait des effectifs plus restreints et de la variance résiduelle relativement élevée dans le cas présent. Ce résultat suggère néanmoins une supériorité moyenne de la descendance des mères hétérozygotes. Une comparaison intra-père des deux types de mères semble le confirmer.

Aucun autre caractère ne présente de différence s'approchant du seuil 5 p. Ioo de signification.

La différence de ponte suggérée entre filles de mères $\mathrm{Hi} H i$ ou $\mathrm{Hi}$ hi pourrait s'interpréter par une supériorité du génotype hétérozygote $H i$ hi sur l'homozygote $H i H i$, ce dernier étant en proportion moindre dans la descendance des mères $H i$ hi.

Cependant, une autre hypothèse difficile à écarter a priori serait celle d'un " effet maternel " favorable exercé par les mères $H i$ hi. Il n'était pas possible de 
comparer des croisements réciproques incluant des mères $H i$ hi d'une part, $H i H i$ de l'autre. Nous avons pu, du moins, comparer la ponte de filles issues d'accouplements du type ơ $H i h i \times$ o $h i h i$ et ơ $h i h i \times+H i h i$. Seules, deux années (I968 et I969) contenaient en même temps ces croisements réciproques, et les effectifs correspondants étaient peu nombreux : ro mères et 80 filles au total pour le premier croisement, II mères et I2I filles pour le second. Les nombres moyens d'œufs sont respectivement 83,6 pour les filles issues du premier croisement (mères $h i$ hi) et 87,6 pour celles de mère $H i$ hi. Il semblerait donc à première vue y avoir un effet favorable des mères $H i$ hi, comparées du moins aux $h i$ hi; mais les données sont trop restreintes et une analyse de variance (tab1. 6) montre que la différence n'est pas significative.

\section{TABLEAU 6}

Analyse de variance du nombre d'cufs:

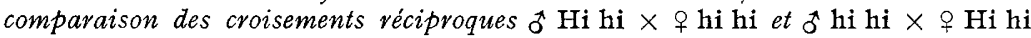

\begin{tabular}{|c|c|c|c|c|c|c|c|c|c|c|c|}
\hline Source & de & vari & iat & io & 1 & (1) & & $\begin{array}{c}\text { Degrés } \\
\text { de liberté }\end{array}$ & Variance & $\mathrm{F}$ & Signification \\
\hline $\begin{array}{l}\text { Croisement } \\
\text { Année . } \\
\text { résiduelle . }\end{array}$ & $\begin{array}{l}\cdot \\
\cdot \\
\cdot\end{array}$ & $\begin{array}{ll}\cdot & \cdot \\
\cdot & . \\
. & . \\
& .\end{array}$ & $\begin{array}{l}. \\
. \\
. \\
.\end{array}$ & & & .. & $\begin{array}{l}\cdot \\
. \\
.\end{array}$ & $\begin{array}{r}\mathrm{I} \\
\mathbf{I} \\
\mathrm{I} 6\end{array}$ & $\begin{array}{r}69,6 \\
\text { I } 578,0 \\
86,0\end{array}$ & $\begin{array}{r}0,8 \mathrm{I} \\
\mathrm{x} 8,36 \\
\end{array}$ & $\mathrm{P}<$ o, OOI \\
\hline
\end{tabular}

(1) Variance " interaction " non calculée exactement.

\section{DISCUSSION}

\section{I. - Réalité et interprétation des différences constatées}

La différence la plus importante et la plus significative est celle entre poules " positives " et " négatives" pour le facteur $H i$, concernant le nombre d'œufs obtenus pendant les premiers mois de production.

Cette différence ne peut être un artefact provenant du fait connu (ScHEINBERG et RECKEL, I96Ic) que le facteur $H i$ ne s'extériorise que chez les poules en ponte. En effet, comme signalé plus haut, la grande majorité des individus pondaient à moins de deux semaines d'intervalle de la date des deux analyses ou au moins de l'une d'elles; les poules trouvées négatives et ne remplissant pas cette condition n'étaient pas prises en considération. Le risque d'avoir classé " négatives " des femelles possédant le facteur $H i$ mais ne l'ayant pas manifesté doit ainsi être très faible : on ne peut estimer à plus de I à 2 p. Ioo au maximum la proportion de " négatives " n'ayant pas pondu à une date très rapprochée d'un test et dont le classement pourrait à la rigueur poser une question, et même en supposant leur ponte très inférieure à la moyenne, cela ne pourrait produire qu'une faible différence - certainement moins de I p. Ioo - entre les nombres moyens d'œufs pour 
les deux phénotypes enregistrés. Les proportions satisfaisantes des ségrégations paraissent d'ailleurs en être une confirmation indirecte, et aussi le fait que, sur plus de I5O poules notées "négatives ", issues de familles présentant une ségrégation et ayant reproduit par la suite, aucune ne s'est révélée posséder l'allèle $H i$ d'après sa descendance.

Par contre, un petit nombre de femelles notées " positives " et. n'ayant pas pondu durant les mois où les tests étaient réalisés n'ont pas été écartées de l'analyse, ce qui pourrait créer une très légère distorsion — pratiquement négligeable aussi - mais cette fois en sens inverse du résultat observé.

Concernant, d'autre part, les différences moins importantes mais significatives observées pour d'autres caractères (poids adulte, épaisseur de coquille, hauteur de 1'albumen, variance de l'âge au I $^{\text {er }}$ œuf), on ne voit pas de possibilité de distorsion du fait de notre mode d'analyse.

On peut donc conclure que, dans notre troupeau et nos conditions d'élevage, les poules possédant au moins un gène $H i$ ont une production d'œufs supérieure, du moins en début de ponte, une maturité sexuelle plus groupée dans le temps, un poiđs légèrement inférieur, leurs œufs ayant une coquille un peu plus épaisse et un albumen moins consistant.

Nos résultats suggèrent en outre qu'il s'agit vraisemblablement, pour la ponte, d'une supériorité de l'hétérozygote $H i$ hi sur les deux homozygotes, quoique 1'hypothèse d'un effet favorable associé à la présence du gène $H i$ chez la mère ne puisse être éliminée totalement pour l'instant.

Cet avantage attaché à l'état hétérozygote pour un gène responsable d'une différence antigénique serait analogue à ceux déjà signalés pour des gènes de groupe sanguin chez la poule (par exemple BRILES, I960; GILMOUR, I960). On peut aussi remarquer - quoique les données actuelles soient encore fragmentaires - la fréquence du polymorphisme signalé pour ce facteur dans la littérature et sa présence notamment dans des lignées consanguines (ERDös, I969) qui peuvent faire soupçonner un avantage sélectif global de 1'hétérozygote. En accord possible avec cette hypothèse, on peut signaler en outre que, sur nos données, le nombre de reproductrices $H i H i$ et $H i h i$ issues de croisements o $H i h i \times$ o $H i h i$ est respectivement, au total, de II et 35 (nombres théoriques $I_{5}, 3$ et 30,7); les nombres correspondants issus du croisement o $H i h i \times$ o $H i H i$ sont 2 et 8 : dans les 2 cas, malgré les petits nombres, on peut noter qu'il y a un excès des o우 hétérozygotes parmi les reproductrices, sans qu'un choix intentionnel ait été possible.

Il reste à discuter maintenant une contradiction au moins apparente entre notre résultat et celui d'un groupe d'autres auteurs.

\section{2. - Comparaison avec les résultats d'autres auteurs}

Nous avons mentionné au début de cet article que PETrovsky et al. (I968) observent une ponte supérieure pour des poules Leghorn n'extériorisant pas le facteur $H i$ testé après dix mois de production.

Ces auteurs suggèrent une corrélation de nature physiologique avec l'état 
de ponte, plutôt qu'une association entre le génotype pour $H i$ et la production d'œufs. De fait, les génotypes de leurs animaux ne paraissent pas — du moins dans leur article - avoir été vérifiés par "progeny-test".

Les poules "physiologiquement " non-réagissantes lors du test pour le facteur $H i$ sont précisément celles qui ne se trouvaient pas en ponte à cette époque. Pour expliquer une ponte supérieure de ces poules pendant la période de contrôle, il faudrait supposer l'existence d'une corrélation négative appréciable entre la production durant cette période et la ponte lors du test pour $H i$. Ceci est a priori possible, divers auteurs (par exemple Gowe et Strain, I963; Nordskog, Festing et VERGHESE, I967) ayant observé une corrélation négative entre le nombre d'œufs obtenus en début et en fin de période de production.

Dans cette hypothèse, nos résultats ne seraient pas nécessairement contradictoires avec ceux des auteurs cités, une supériorité du génotype $\mathrm{Hi}$ hi (ou $\mathrm{Hi} \mathrm{Hi}$ ) sur $h i$ hi en début de ponte n'empêchant pas que celles des poules du premier génotype apparues "physiologiquement" négatives après dix mois de ponte aient pu être les meilleures pondeuses auparavant.

Dans l'hypothèse, toutefois difficile à exclure définitivement, où dans les données de Petrovoskx et al., les meilleures pondeuses auraient correspondu au génotype $h i$ hi, plusieurs différences existent avec nos résultats. Tout d'abord, nous enregistrons la période de début de ponte, en moyenne les cinq premiers mois (pour nos poules écloses en mars-avril, l'entrée en ponte se situait entre juillet et septembre et le contrôle s'arrêtait fin décembre) au lieu de dix mois pour les auteurs cités : il faudrait vérifier si le facteur $H i$ peut exercer une influence sur la répartition de la production entre la première et la seconde moitié de l'année de ponte.

On pourrait aussi concevoir que le facteur $H i$ soit en réalité lié à un gène influant sur l'intensité de ponte, en sens contraire dans notre troupeau et chezles Leghorns de Petrovsky et al., ou encore que son expression soit modifiée par une interaction avec le reste du génôme ou les conditions de milieu.

D'autres recherches sont nécessaires pour tirer une conclusion sur ces divers points : la relative importance des différences que nous trouvons associées à ce facteur nous paraît largement justifier leur intérêt. Concernant en particulier 1'hypothèse qui nous est suggérée d'une supériorité de l'état hétérozygote à ce locus pour la ponte, il serait intéressant de vérifier systématiquement dans quelle mesure on observe le maintien non aléatoire d'un polymorphisme pour ce gène dans des lignées consanguines.

Reçu pour publication en octobre r97o.

\section{SUMMARY}

\section{Hi AGGLUTINOGEN AND PERFORMANCES IN POULTRY}

Having observed in our Poultry flock a polymorphism for an agglutinogen present un red blood cells and detected by seed extracts (Lens culinaris), we verified that it is transmitted as a single gene with two alleles, the presence of the agglutinogen being dominant. In view of its 
characteristics, we designate this gene by the symbol $\mathrm{Hi}$ already known (ScHEINBERG and RECKEL, 1961).

We looked for quantitative differences possibly associated to this factor. In our population, " positive" hens ( $\mathrm{Hi} / \mathrm{Hi}$ or Hi/hi) lay 8.5 per cent more eggs than " negative" ones (hi/hi) during the first months of production $(\mathrm{P}<.001)$. This cannot reasonably be attributed to an absence of expression of the agglutinogen among females which are out of laying, and shoulá thus represent a real difference associated to the compared genotypes. On the other hand, the variances of egg number and age at first egg are lower for "positive" hens $(P<.01)$, their eggshells are slightly thicker and less variable $(\mathrm{P}<.05)$, the albumen of their eggs is less consistent $(\mathrm{P}<.001)$. Finally, their adult weight is $40 \mathrm{~g}$ inferior $(\mathrm{P}<.05)$. On the contrary, there is no difference of mortality between the animals of the two phenotypes.

An indirect comparison between the $\mathrm{Hi} / \mathrm{Hi}$ and $\mathrm{Hi} / \mathrm{hi}$ genotypes suggests a superiority of the heterozygote over the two homozygotes for egg number.

\section{RÉFÉRENCES BIBLIOGRAPHIQUES}

BOREL J. F., I962. The usefulness of lectins for detecting individual differences among chickens. Vox Sang., 7, 632-637.

Borel J. F., I964. Recherches immunogénétiques sur des substances spécifiques de groupes chez la poule et sur leur utilisation comme marqueurs de gènes dans l'élevage. Thèse, Zurich.

BrILES W. E., I960. Blood groups in chickens, their nature and utilization. Worlds Poultry Science Journal, 16, $223-242$.

ERDÖs A., 1969. Untersuchungen mit Phytoagglutininen bei Inzuchthühnern. Blut, 19, 559-563.

Gilmour D. G., I96o. Blood groups in chickens. Brit. Poult. Sci., 1, 75-1oo.

GowE R. S., STRAIN J. H., 1963. Effect of selection for increased egg production based on part-year. records in 2 strains of white L,eghorns. Can.J. Genet., Cytol., 5, 99-Ioo (abstr.).

MÉrat P., I967. Contribution à l'étude de la valeur sélective associée à quelques gènes chez la poule domestique. Ann. Biol. anim. Bioch. Biophys., 7, 79-104.

Mrrsumoto $T$., I964. Chicken red cell agglutinogens and plant lectins. Immunogenetics Letters 3.

Nordskog A. W., Festing M., Verghese M. W., I967. Selecting for egg production and correlated responses in the Fowl. Genetics, 55, r79-r9r.

Petrovsky E., Macha J., Soutorova J., Vondreje J., r968. Genetics of immunological and physiological characters in poultry. V. Phenotype frequency variations in the erythrocyte $H i$ system of chickens with selection for characters of economic importance. Acta Univ. agric., Brno, 16, 667-672.

Scheinberg S. I., RECkEL R. P., I96ra. Detection of red cell agglutinogens with lectins in chicken. Poult. Sci., 40, 689-698.

ScheINBerg S. I., REckel. R. P., r $96 \mathrm{t} b$. Somatic variation of red cell agglutinogens due to hormonal in fluence. Poult. Sci., 40, 795-807.

Scheinberg S. I., REckel R. P., I961c. Fffect of œstrogen on somatic variation of an erythrocyte agglutinogen in chickens. Nature, 189, 295-296.

Schennberg S. I., ReCkel R. P., I962. Studies on the ",Hi " agglutinogen in chickens. Ann. N.Y. Acad. Sci. 97, 194-204. 